\title{
miR-486-5p expression is regulated by DNA methylation in osteosarcoma
}

\author{
Heidi M. Namløs', Magne Skårn', Deeqa Ahmed², Iwona Grad', Kim Andresen², Stine H. Kresse', \\ Else Munthe ${ }^{1}$, Massimo Serra ${ }^{3}$, Katia Scotlandi ${ }^{3}$, Antonio Llombart-Bosch ${ }^{4}$, Ola Myklebost ${ }^{1,5}$, Guro E. Lind ${ }^{2}$ and \\ Leonardo A. Meza-Zepeda ${ }^{1,6^{*}}$
}

\begin{abstract}
Background: Osteosarcoma is the most common primary malignant tumour of bone occurring in children and young adolescents and is characterised by complex genetic and epigenetic changes. The miRNA miR-486-5p has been shown to be downregulated in osteosarcoma and in cancer in general.

Results: To investigate if the mir-486 locus is epigenetically regulated, we integrated DNA methylation and miR486-5p expression data using cohorts of osteosarcoma cell lines and patient samples. A CpG island in the promoter of the ANK1 host gene of mir-486 was shown to be highly methylated in osteosarcoma cell lines as determined by methylation-specific PCR and direct bisulfite sequencing. High methylation levels were seen for osteosarcoma patient samples, xenografts and cell lines based on quantitative methylation-specific PCR. 5-Aza-2'-deoxycytidine treatment of osteosarcoma cell lines caused induction of miR-486-5p and ANK1, indicating common epigenetic regulation in osteosarcoma cell lines. When overexpressed, miR-486-5p affected cell morphology.
\end{abstract}

Conclusions: miR-486-5p represents a highly cancer relevant, epigenetically regulated miRNA in osteosarcoma, and this knowledge contributes to the understanding of osteosarcoma biology.

Keywords: miRNA, Bone, cancer, Cell line, Epigenetic, Sarcoma, ANK1

\section{Introduction}

High-grade osteosarcoma is the most prevalent primary malignant tumour of bone, affecting both children, adolescents and, more rarely, elderly people. Following the introduction of multi-agent chemotherapy in the 1970s, the 5-year survival rate increased considerable reaching $60-70 \%$ among patients with conventional high-grade osteosarcoma [1]. However, advances in treatment have stalled with no further improvement in the survival [2], and also exhibit a collateral risk for adverse toxicity events. Improved biological knowledge is required

\footnotetext{
*Correspondence: Leonardo.Meza-Zepeda@rr-research.no

${ }^{6}$ Genomics Core Facility, Department of Core Facilities, Institute

for Cancer Research, The Norwegian Radium Hospital, Oslo University

Hospital, Oslo, Norway

Full list of author information is available at the end of the article
}

to develop new treatment opportunities and further improve the survival of osteosarcoma patients.

Osteosarcoma is characterized by considerable phenotypic and genomic heterogeneity, and few recurrent targetable genetic changes have been reported. Osteosarcoma exhibits a complex karyotype with high genetic and chromosomal instability seen as multiple rearrangements across the genome, kataegis and chromothripsis [3-6]. The genetic markers identified have been associated with treatment response and prognosis, thus appearing as promising candidates for a translation to clinical practice $[7,8]$. However, the limited extent of recurrent profiles identified indicates that a substantial regulation of the transcriptional programs in osteosarcoma may rather be caused by epigenetic programs [9-13], providing novel avenues for cancer therapy [14]. 
Epigenetic mechanisms are fundamental drivers of tumour initiation, development and progression [15]. Several epigenetic alterations have been identified, including biomarkers for various diseases [16]. DNA methylation is the most commonly studied epigenetic alteration in cancer [17], comprising covalent addition of methyl groups to CpG sites. CpG islands (CGIs) are characterized by regional enrichment of $\mathrm{CpG}$ sites and are present in approximately $70 \%$ of all human gene promoters [18]. In normal cells, these CGIs are usually unmethylated. In cancer, however, CGI hypermethylation is frequently observed, accompanied by long-term silencing of gene expression [19].

MicroRNAs (miRNAs) are a key class of epigenetic regulators as they act to post-transcriptionally silence large numbers of genes without modifying the DNA [20]. miRNAs are frequently associated with CGIs and are themselves also found silenced through epigenetic mechanisms [21]. Dysregulated miRNA expression may result in aberrant expression of genes that play critical roles in osteosarcoma tumorigenesis and progression [22-25]. We have previously identified miR-486-5p to be among the most downregulated miRNAs in osteosarcoma cell lines [24]. miR-486 has been shown to be downregulated in osteosarcoma patient samples compared to normal samples [26]. However, the mechanism of repression of miR-486-5p is still unknown in osteosarcoma. Given that epigenetic regulation of miR-486-5p has been described in other cancers like lung cancer [27], we hypothesized that this could be the mechanism of miR-486-5p regulation also in osteosarcoma.

In an effort to advance the understanding of osteosarcoma biology, we aimed to investigate if the expression of miR-486-5p was epigenetically regulated through methylation of promoter regions. We analysed miR-486-5p expression and DNA methylation levels in a cohort of osteosarcoma cancer cell lines and patient samples. Qualitative and quantitative methylation analyses were performed, allowing us to characterize the mir-486 locus in detail. Finally, the in vitro effect of miR-486-5p overexpression on cell morphology and proliferation was investigated.

\section{Results}

\section{Low expression of miR-486-5p in osteosarcoma}

To follow up on our earlier observations of miR-486-5p in osteosarcoma [24], miR-486-5p expression level was examined in a panel of osteosarcoma patient samples $(n=9)$, osteosarcoma cell lines $(n=17)$ and normal bone samples $(n=6)$ by quantitative real-time reversetranscription PCR (qRT-PCR). The mean expression of miR-486-5p was reduced 3-fold in patient samples $(p<0.01)$ and 300 -fold in cell lines $(p<0.0001)$ compared to normal bone (Fig. 1a). This confirms a low expression of miR-486-5p in osteosarcoma using both patient tissue samples and cell lines.

\section{DNA methylation is associated with low expression of miR-486-5p in osteosarcoma cell lines}

miRr-486-5p is encoded within the last intron of the Ankyrin 1 (ANK1) gene. Several transcript variants of human ANK1 exist, with multiple transcriptional start sites (TSS), promoter regions and CGIs. The ANK1 variants 1-4 represents medium-sized transcripts, $A N K 1$ variant 9 is a longer transcript while $S A N K 1$ represents the short variants 5, 7 and 10 (Additional file 1: Supplementary Fig. S1). The methylation level of ANK1 was quantified in osteosarcoma cell lines $(n=19)$ and normal bone samples $(n=4)$ using Illumina HumanMethylation27 BeadChips. This microarray contains one CpG site in the CGI CpG79 (chr8:41654876-41,655,984) at the TSS (position chr8:41655140) of ANK1 (variant 1-4). The average difference of methylation in cell lines versus normal bone samples was 0.36 (DeltaBeta) for CpG79, providing a first evidence for methylation of the mir-486 locus.

We next compared the expression and methylation level of miR-486-5p for the above sample cohort. The expression level of miR-486-5p was quantified using Agilent miRNA array v2. The mean expression of miR486-5p was significantly lower in cell lines than in bone $(p<0.0001)$ and the methylation was higher in cell lines than in bone $(p<0.01)$. Taken together, this indicated an association between low expression of miR-486-5p and CpG methylation of ANK1 (Fig. 1b).

Demethylation caused increased expression of miR-486-5p We next examined the effect of changes in methylation on miR-486-5p expression. Osteosarcoma cell lines $(n=12)$ were treated with the demethylation agent 5-Aza-2'-deoxycytidine (5-Aza). After $72 \mathrm{~h}$, the relative expression levels of miR-486-5p in untreated and treated cell lines were quantified by qRT-PCR. A significant difference between the cells before and after treatment was observed $(p=0.02)$. miR-486-5p showed at least a 1.3 -fold (30\%) induction in $8 / 12$ tested cell lines (Fig. 2a), indicating that the expression of miR-486-5p was affected by changes in DNA methylation.

To exclude that the expression level of mature miR486-5p was affected by post-transcriptional processing, the pri-miRNA hsa-mir-486-1 (pri-mir486) was quantified by qRT-PCR. pri-mirR486 showed at least 1.3 fold 

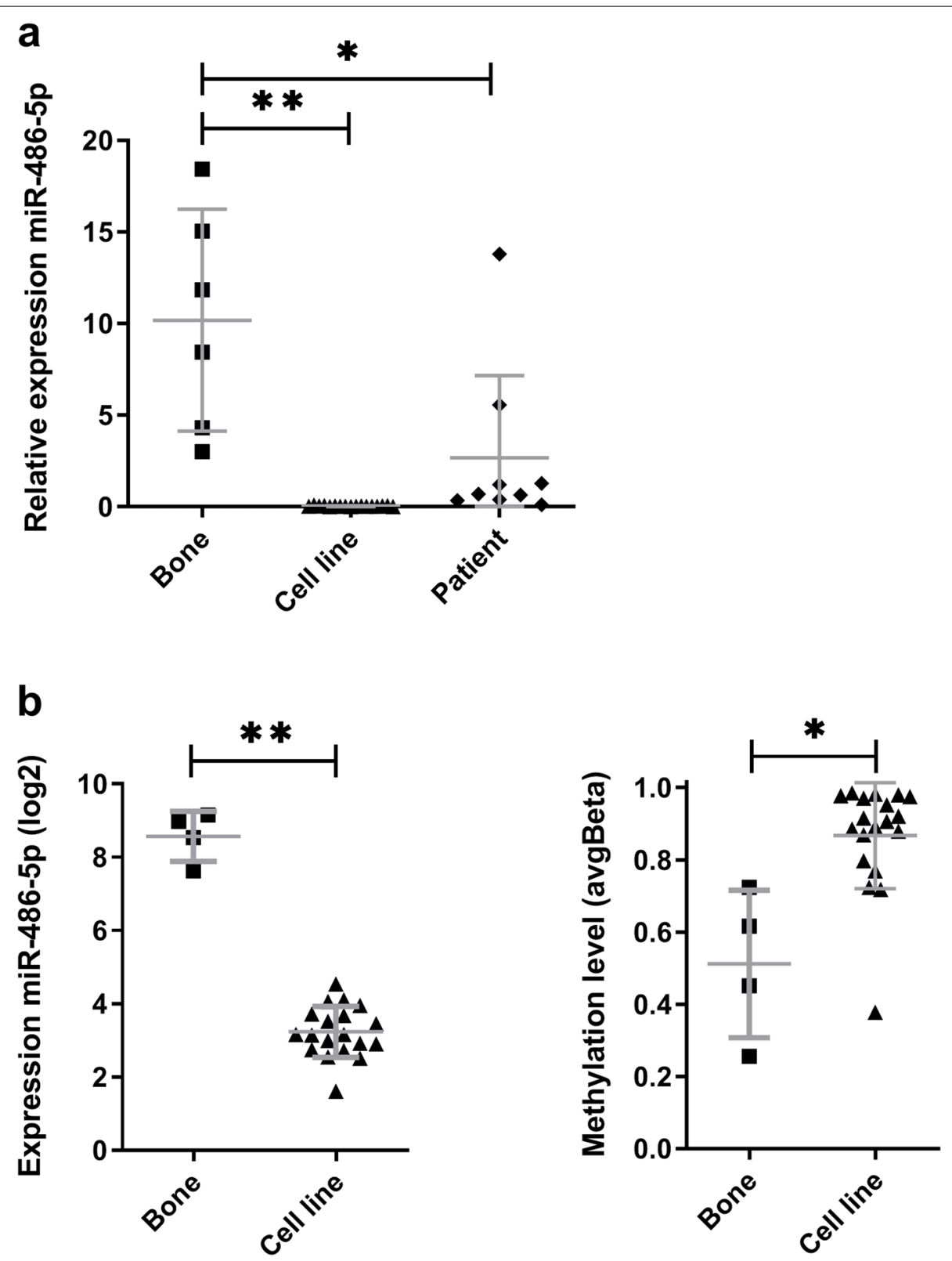

Fig. 1 miR-486-5p expression and methylation in osteosarcoma cell lines and patient samples. A. miR-486-5p expression level in normal bone $(n=6)$, cell lines $(n=17)$ and patient samples $(n=9)$ using qRT-PCR. The expression level was quantified for the groups of samples. The values are shown relative to mean expression of bone. B. DNA methylation and miRNA expression levels in normal bone samples $(n=4)$ and osteosarcoma cell lines $(n=19)$ using arrays. The expression level of miRNAs (log2) and DNA methylation level (Beta, probe cg00176210) were obtained using Agilent miRNA array v2 and Illumina Infinium Methylation27 BeadChip technology, respectively. Values are given as mean (SD) with whiskers from min to max. $P$-values ${ }^{*}<0.01$ and ${ }^{* *}<0.0001$

(30\%) induction in 9/12 cell lines after 5-Aza treatment, confirming the epigenetic effects seen on the mature miRNA (Fig. 2b). A high correlation with was observed between mature miR-486-5p and the pri-miRNA, with Pearson's correlation $\mathrm{r}=0.97(p<0.0001)$ before 5-Aza treatment and $\mathrm{r}=0.92(p<0.0001)$ after treatment of the cell lines (Fig. 2c and d).

\section{The genes within the mir-486/ANK1 locus were observed} to be co-regulated

To address the relationship between expression of miR-486-5p and the different $A N K 1$ transcript variants, transcript-specific qRT-PCR was performed. The human $A N K 1$ variant 9 has the most upstream first exon, whereas an alternative exon 1 is used in variants $1-4$. 


\section{a}

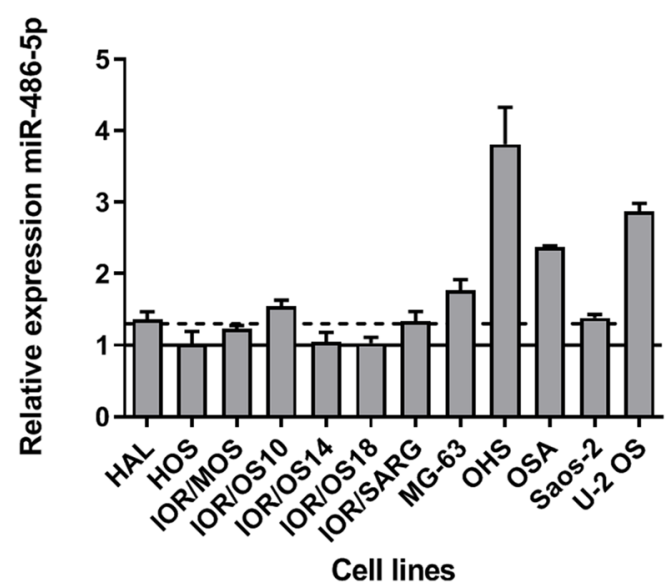

C

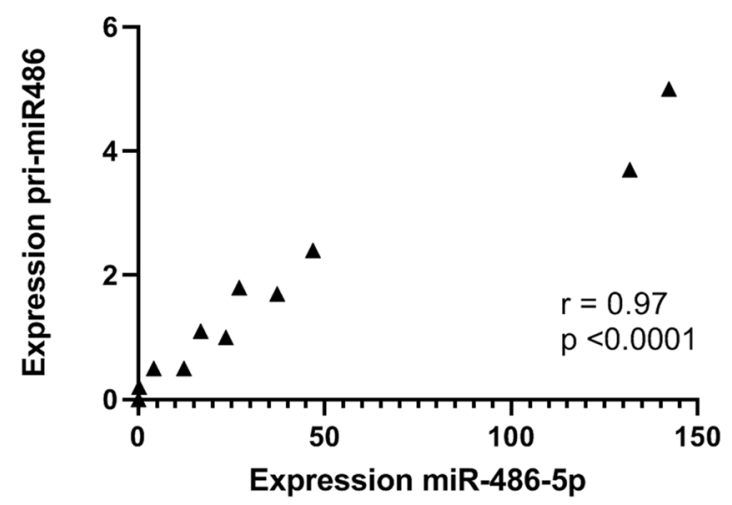

b

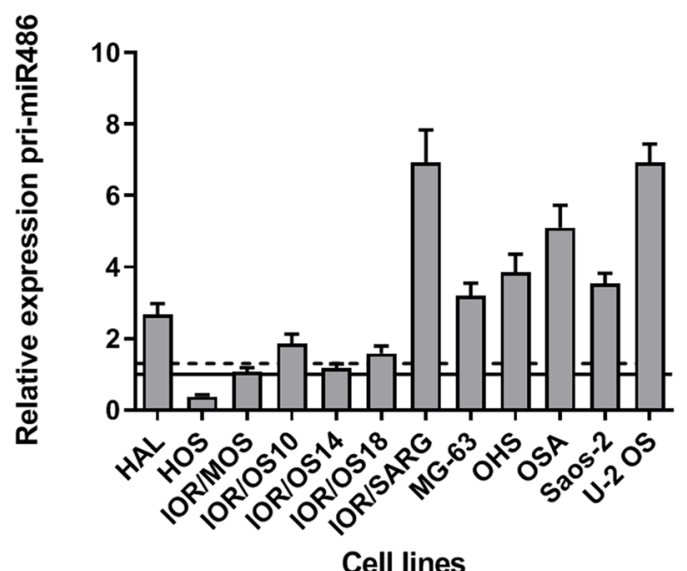

d

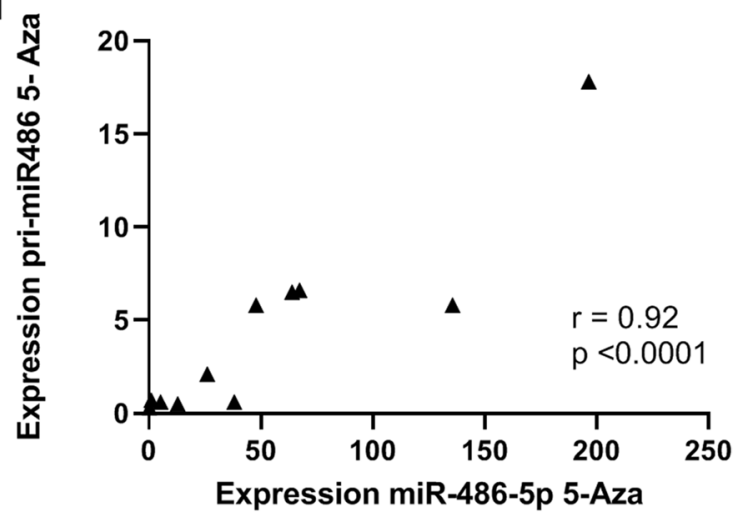

Fig. 2 Expression and correlation of miR-486-5p and hsa-mir-486-1 (pri-mir486) in osteosarcoma cell lines upon 5-Aza-2'-deoxycytidine treatment. Relative expression level A. miR-485-5p and B. pri-mir486 after $72 \mathrm{~h}$ of 5-Aza treatment. The values are shown relative to treated cell lines (set to 1 , horizontal line). Induction of $>30 \%$ shown as dotted line. Values are given as mean (SD). Correlation between expresion level of miR-485-5p and pri-mir486 in C. untreated and D. 5-Aza treated cell lines. The expression levels are quantified using qRT-PCR, and normalized against RNU44 for miR-486-5p and GAPDH for pri-mir486

$s A N K 1$ represents the short variants 5,7 and 10 , with an alternative exon 1, while exons 41 and 42 are in common with the other transcript variants (Additional file 1: Supplementary Fig. S1).

In osteosarcoma cell lines, $A N K 1$ variants $1-4$ were found to be moderately expressed in 8/12 lines. The $s A N K 1$ variant was only weakly detected in $3 / 12$ cell lines, while variant 9 was undetected (Additional file 1: Supplementary Fig. S2). Comparing expression levels of miR-486-5p and $A N K 1$, no similarity was observed between miR-486-5p and sANK1 or ANK1 variant 9. For miR-486-5p and ANK1 variants 1-4, a high correlation (Pearson's correlation $\mathrm{r}=0.93, p<0.0001$ ) could be observed across the cell line panel if one of the cell lines, MG-63, was omitted (Fig. 3a). Similar observations were done for pri-miR486 (Pearson's correlation $r=0.92$, $p<0.0002)$. The cell line MG-63 showed very high expression levels of $A N K 1$ variants 1-4, however as the sample seems to be a biological and not a technical outlier, it was not removed from the dataset.

ANK1 variant 1-4 expression was induced in 8/12 cell lines upon 5-Aza treatment (Additional file 1: Supplementary Fig. S2). Comparing the effect of 5-Aza treatment on the mRNA level and miRNA level, it was observed that all 7 cell lines with increased expression of miR-486-5p also had increased expression of $A N K 1$ variant 1-4. The level of induction was similar for $A N K 1$ and miR-486-5p, and a high correlation (Pearson's Correlation $r=0.93, p<0.0001)$ could be seen between the fold change observed for $A N K 1$ and the fold change observed for miR-486-5p upon Aza treatment across all 12 cell lines (Fig. 3b). No correlation between miR-486-5p and 

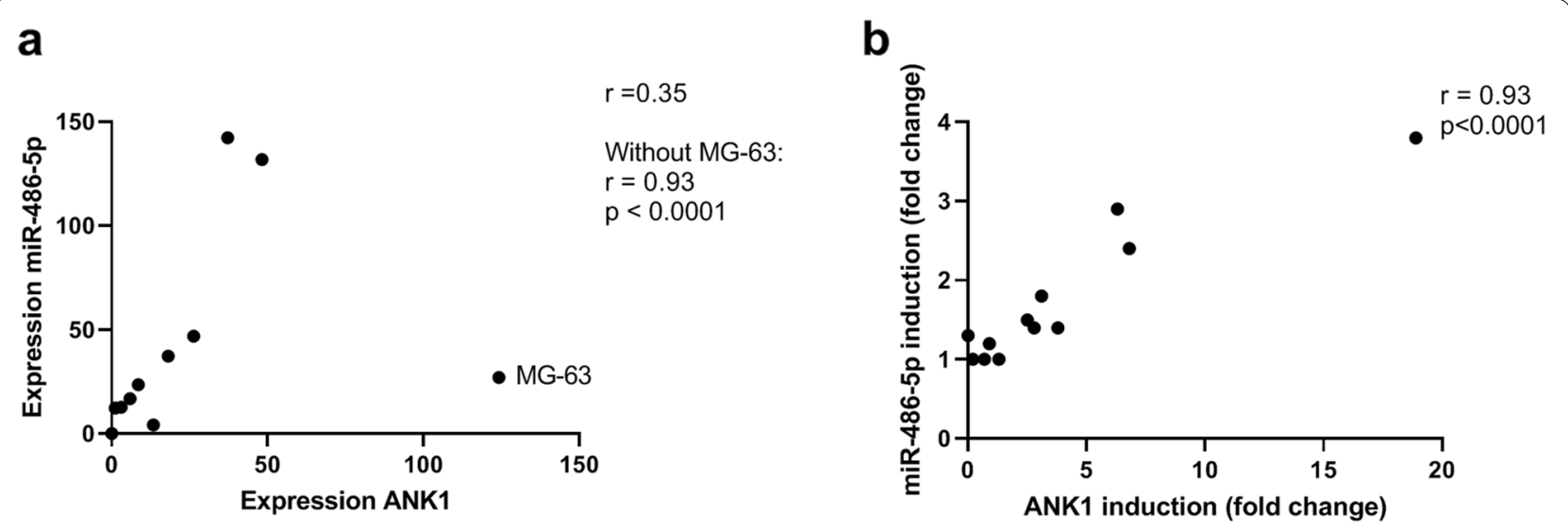

Fig. 3 Expression and correlation of miR-486-5p and ANK1 before and after 5-Aza-2'-deoxycytidine treatment in osteosarcoma cell lines. A. Quantification of expression of miR-486-5p and ANK1 versions 1-4 in untreated cells. B. The induction of miR-486-5p and ANK1 versions 1-4 after 5-Aza treatment. The induction fold change is calculated as the ratio between expression of untreated and 5-Aza treated transcripts as quantified by qRT-PCR. The expression is normalized against RNU44 for miR-486-5p and GAPDH for ANK1. Pearson's Correlation $r$ is calculated with and without the outlier sample MG-63

$A N K 1$ induction was observed for the other $A N K 1$ variants. The $s A N K 1$ variant was only induced in four cell lines and no variant 9 transcripts could be detected upon 5-Aza treatment (Additional file 1: Supplementary Fig. $\mathrm{S} 2$ ). These observations support a co-regulation between miRNA and mRNA genes in the $m i R-486 / A N K 1$ locus.

\section{Genome-wide methylation profiling revealed hypermethylation of a CGI upstream of mir-486 in osteosarcoma patient samples}

To investigate the methylation level of the whole mir486 locus, we performed high-resolution Infinium $450 \mathrm{k}$ methylation array analysis on a set of 10 osteosarcoma patient samples and four normal bones. For the mir-486/ ANK1 locus, methylation data from 96 CpG sites across all CGIs were determined. The CpG sites located in nonCGI regions were in general hypermethylated in both groups of samples, with a higher level for bone. Higher methylation levels for osteosarcoma patient samples compared to bone were only observed in the CGI CpG79 (Fig. 4a), showing different levels of methylation within the patient cohort. The bone samples show a similar pattern of methylation across the CGIs, but with lower level of methylation with only partially methylated and unmethylated sites (Fig. 4b).

The patients samples were classified as unmethylated or methylated based on methylation level of one of the CpG sites that had the most evident methylation in CGI CpG79. In general, miR-486-5p was observed to be low expressed in highly methylated osteosarcoma samples and higher expressed in unmethylated samples with $p=0.03$. The bone samples showed the same level of expression as unmethylated osteosarcoma samples
(Fig. 4c). This suggests that methylation of an upstream regulatory region affects miR-486-5p expression in osteosarcoma patients.

Qualitative and quantitative methylation analysis confirms hypermethylation of mir-486 in osteosarcoma

Qualitative methylation-specific polymerase chain reaction (MSP) analysis was done for CGI CpG79 and CpG171, both located upstream of mir-486. The CGI CpG79 was hypermethylated in 20/21 cell lines (Fig. 5). Interestingly, this CGI was unmethylated in the IOR/ OS14 cell line, previously shown to be globally hypomethylated [10]. The CGI CpG171 was only found to be hemimethylated in one cell line, and unmethylated in all the others (Additional file 1: Supplementary Fig. S3). Thus, further focus was done on the CGI CpG79.

Direct bisulfite sequencing was performed on osteosarcoma cell lines. The analysis was done on an extended region of the CGI CpG79, covering a total of $34 \mathrm{CpGs}$ including the region for the MSP on CpG79 (Fig. 5). The resulting data confirmed the methylation status previously determined by MSP.

The methylation of a region of CpG79 of mir-486 was analysed quantitatively by quantitative methylationspecific polymerase chain reaction (qMSP) in a larger cohort of samples, including osteosarcoma cell lines $(n=20)$, xenografts $(n=41)$, patient samples $(n=14)$ and bone $(n=5)$. Methylated percent of methylated reference $(P M R)$ was set to $>5.2$. High qMSP methylation pattern was only observed in cancer samples, comprising both cell lines, xenografts and patient samples (Fig. 6). Mann-Whitney U test was used to compare the PMR values of the candidate regions in the different 


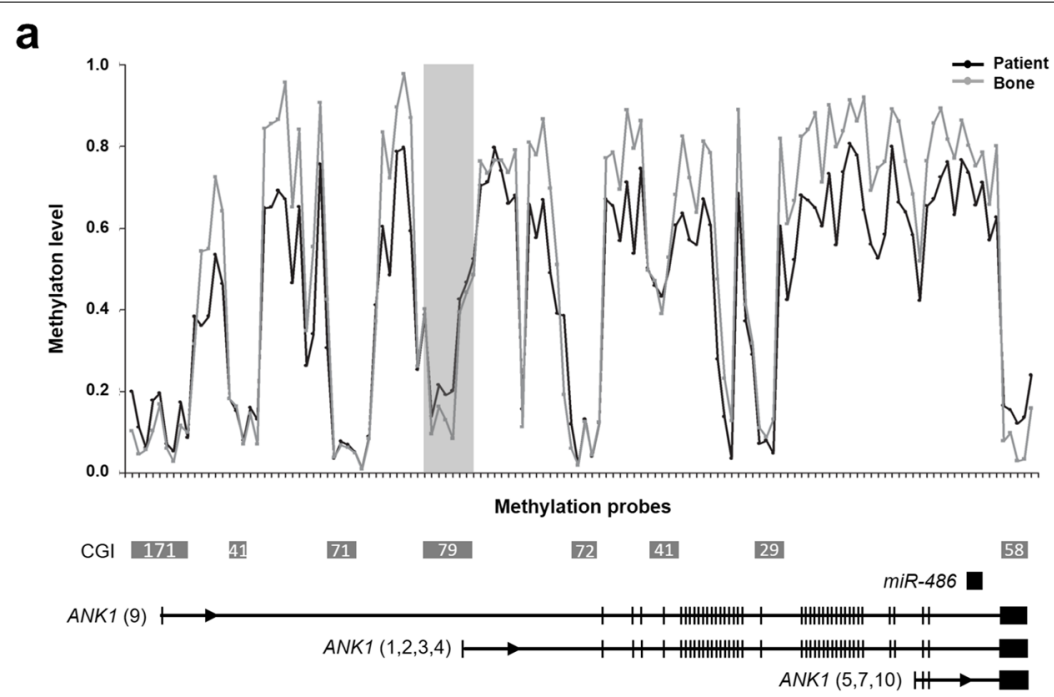

b

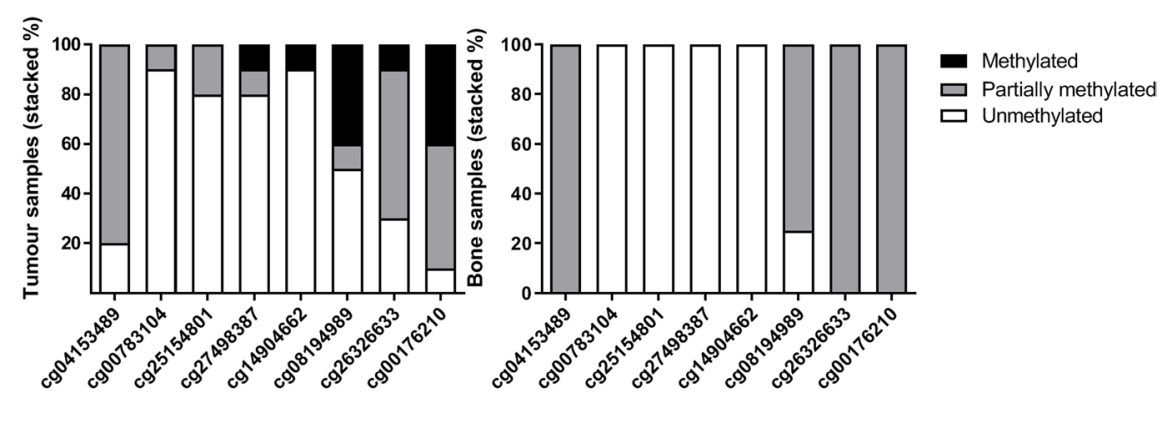

$\mathbf{C}$

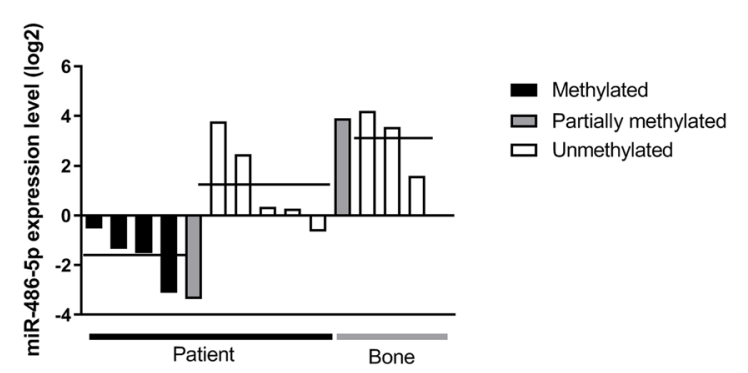

Fig. 4 Genome-wide methylation profile of the mir-486/ANK1 locus and miR-486-5p expression levels in osteosarcoma patient samples. A. Representation of average methylation profile across the mir-486/ANK1 locus in osteosarcoma patient samples $(n=10)$ and bone $(n=4)$. Methylation level (Beta) is shown for the individual CpG sites (ticks on horizontal axis) from the HumanMethylation450 BeadChips. The probes are ordered along the locus and intervals are not in scale. CGls are shown as grey boxes with number refering to CpG count (from UCSC Genome Browser NCBI, GRCh37/hg19 assembly). Horizontal lines below plot show representative transcript variants of the respective mRNA genes (RefSeq) with exons as vertical bars, not in scale. Shaded vertical box: CGI shown in detail in B. Black, osteosarcomas; Grey, bone. B. Probe level methylation for CGI CpG79 in osteosarcoma patient samples and bone. Methylation levels as determined by Infinium $450 \mathrm{k}$ arrays are given in Beta values for the individual CpG sites (cg). The selected CpGs within CGI CpG79 are highlighted with a shaded vertical box in A. Methylated: Beta 0.7-1.0; partially methylated: Beta 0.3-0.7; unmethylated: Beta<0.3. C. Expression level of miR-486-5p in osteosarcoma patient samples and bone based on qRT-PCR. The osteosarcoma samples are grouped based on methylation status for probe cg08194989 on Infinium $450 \mathrm{k}$ arrays, while the bone samples are shown as one group. Horizontal line: mean value

tissues, showing only significant differences between bone and cell lines $(p=0.005)$. For the cell lines, $17 / 20$ $(85 \%)$ showed increased methylation $(>2 \mathrm{x})$ compared to the bone average. However, increased methylation was also observed for $8 / 14(60 \%)$ of the patient's samples and $17 / 41(40 \%)$ of the xenografts. Together, these observations suggest that the CGI CpG79 is highly methylated in osteosarcoma. 

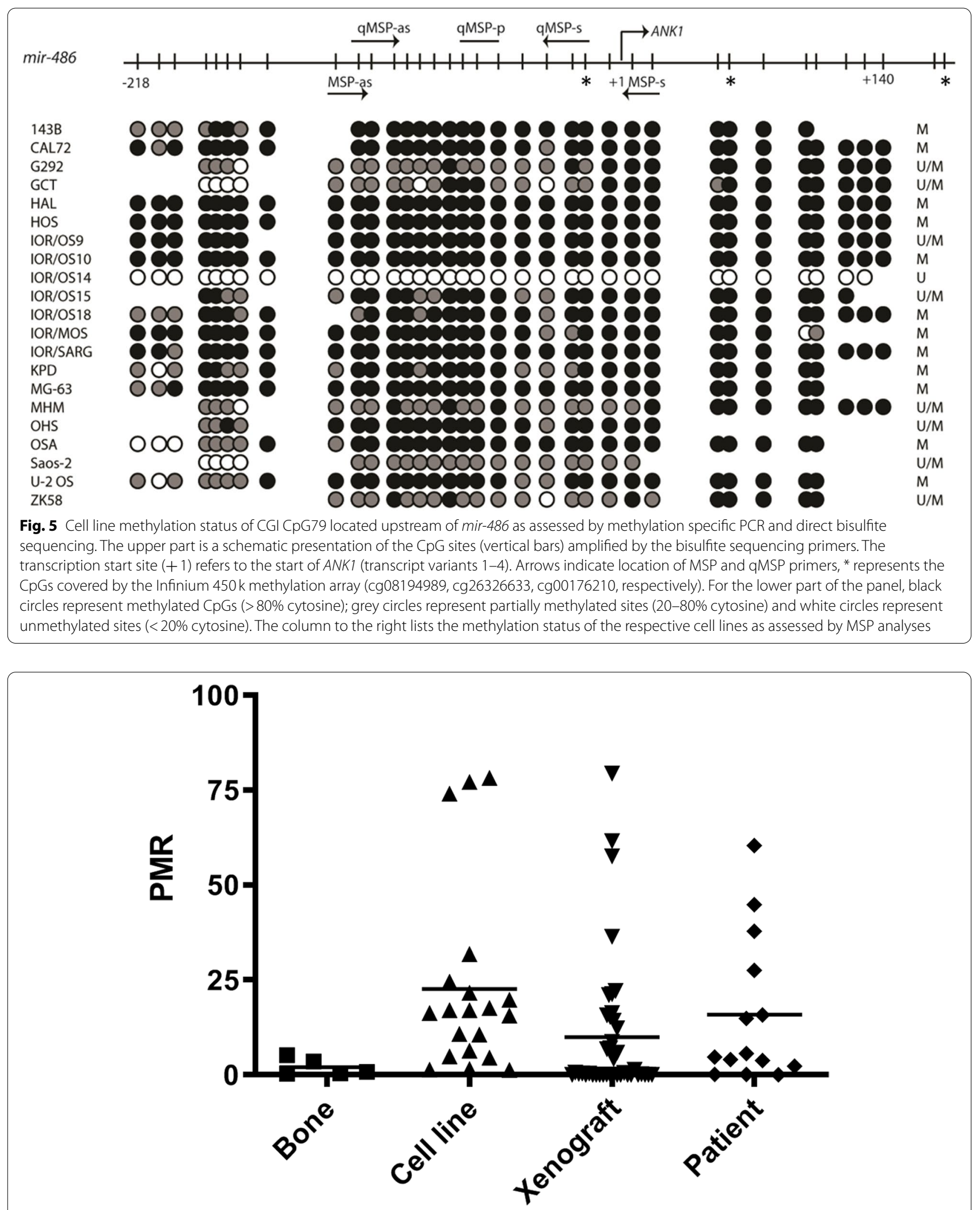

Fig. 6 Methylation distributions in osteosarcoma assessed by quantitative methylation-specific PCR. The methylation was measured for the groups of normal bones and osteosarcoma cell lines, xenografts and patient samples. PMR, percent of methylated reference. Horizontal black line: mean values 


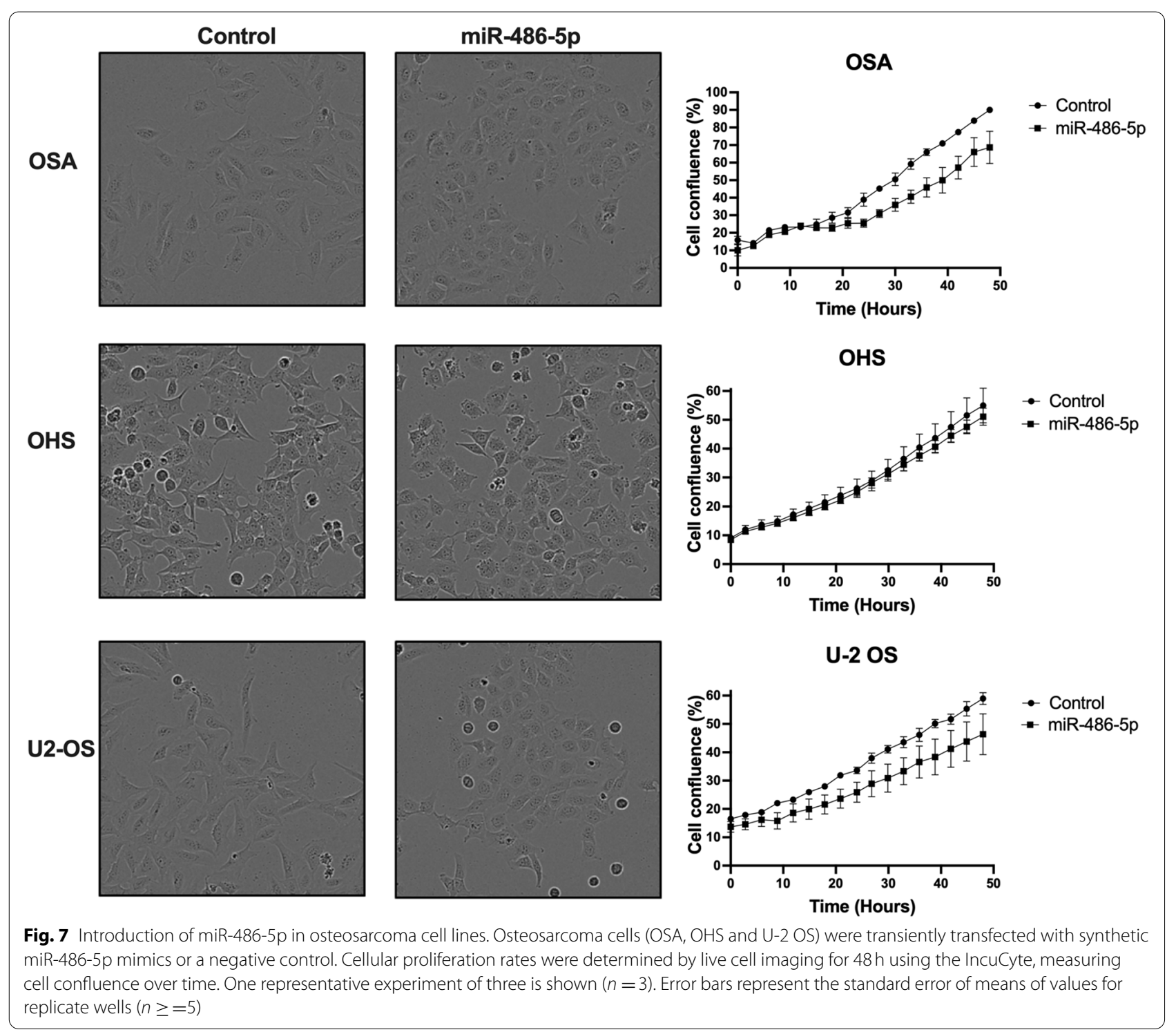

\section{Overexpression of miR-486-5p induces morphological changes in osteosarcoma cell lines}

To investigate the in vitro effect of miR-486-5p overexpression, we transiently transfected the osteosarcoma cell lines OSA, OHS and U-2 OS with miR-486-5p synthetic miRNA mimics. These cell lines showed the highest level of induction upon 5-Aza treatment. The iIntroduction of miR-486-5p caused a reduced growth rate in OSA and U-2 OS at $48 \mathrm{~h}$, although the changes were not statistically significant $(p=0.2)$. However, a clear change in cell morphology was observed in all three cell lines where cells became flatter, rounder and smaller (Fig. 7). Thus, changes in miR-486-5p levels seem to affect the osteosarcoma cell phenotype.

\section{Discussion}

Epigenetic mechanisms such as DNA methylation play crucial roles in controlling miRNA gene expression. Based on our previous observations of a strong, cancerspecific downregulation of miR-486-5p in osteosarcoma cell lines [24], recently confirmed in osteosarcoma patients [26], we set out to investigate the epigenetic regulation of miR-486-5p in osteosarcoma.

In this study, we have shown that the expression of miR-486-5p is differently regulated in osteosarcoma compared to normal bone. A heterogeneity was seen among the patient cohort regarding methylation status. The patients seem to be divided in two groups, one group highly methylated with low expression and a second 
group with an opposite pattern of low methylation and high expression. One might speculate that the miRNA regulation can be associated with disease aggressiveness, but extensive clinical data was not available for all of the patients in the cohort.

mir-486 is located intragenic within the last intron of ANK1, which encodes ankyrin-1. The mir-486/ANK1 locus is complex, and contains multiple promoter regions and CGIs. Alternative splicing and distinct use of promoters give rise to different isoforms of ANK1 [28, 29]. The methylation of the CGI covering the TSS of ANK1 variants $1-4$ indicates that the mir-486 locus is under control of the ANK1 gene promoter in this region. This CGI was the only CGI in this locus that was hypermethylated in a high-resolution analysis, and cancer-specific methylation was verified in cancer cell lines, xenografts and clinical samples. In many cases, intragenic miRNAs are co-expressed with their host genes, suggesting that they depend on the same regulatory mechanisms [30,31]. The similar expression patterns between the pri-miRNA, the mature miR-486-5p and $A N K 1$ variants 1-4 indicate that the miRNA and mRNA of the mir-486/ANK1 locus is transcribed together. Upon demethylation treatment, the expression levels of pri/mature miR-486 and $A N K 1$ changed at similar levels across the osteosarcoma cell line panel, supporting a common epigenetic regulation from the ANK1 variant 1-4 gene promoter.

It has been shown that alternative promoters give individualized regulation of transcripts with distinct first exons, providing an expression pattern with a strong tissue preference [32]. The regulation of the mir-486/ ANK1 locus may depend on the specific cellular context. The putative host gene $A N K 1$ is a prototype of the ankyrin membrane proteins, linking integral membrane proteins to the underlying spectrin network in erythroid cells [33], and has also been found in brain and muscles $[28,34]$. The diversity of the ankyrins suggests that the isoforms might serve different roles in various cell types. The ANK1 variant 1-4, observed to be co-expressed with miR-486-5p in our study, has previously been shown to be relevant for miR-486-5p expression in leukaemia. In myeloid leukaemia with an erythroid phenotype, miR-486-5p was regulated through GATA1 binding to the promoter region of $A N K 1$ variant 1-4 [35]. An early study showed that mir-486-5p can be controlled by an alternative, muscle-specific promoter within intron 40 of the $S A N K 1$ variant [36]. The $S A N K 1$ isoform could only be detected for a few of the osteosarcoma cell lines in our study and was only induced in a subset of the cell lines. An extensive study of miR-486-5p was recently performed in non-small cell lung cancer, showing that miR-486-5p was co-expressed with $A N K 1$ variant 9 in lung cancer and lung epithelial cells. Aberrant methylation of the CGI covering the TSS of $A N K 1$ variant 9 (termed ANK1B promoter) repressed both $A N K 1$ and miR-486-5p [27], and was specific for adenocarcinoma. In our study, this CGI was unmethylated in all the osteosarcoma cell lines and the transcript levels were not induced upon 5-Aza treatment.

The induction of miR-486-5p expression in osteosarcoma cells resulted in a change in cell morphology, as well as a pattern of reduced cell proliferation in 2 out of 3 cell lines tested. A morphological change in MG-63 cells following overexpression of miR-486-5p has been described previously, possibly related to an EMT-like phenotype [37]. Through in vitro experiments, it has been shown that miR-486 can reduce proliferation, promote apoptosis and inhibit metastasis through regulation of the PKC- $\delta$ pathway in osteosarcoma cells [26]. miR-486 also promotes pro-osteogenic activity through induction of myofibroblastic differentiation through the PTEN-AKT pathway [38]. Following our and others observations of a low expression level of miR-485-5p in cancer, miR-486-5p has been described as a tumour suppressor in breast carcinoma, colorectal cancer, oesophagal cancer hepatocellular carcinoma, lung cancer, gastric carcinoma, myxoid liposarcoma, colorectal cancer, oesophagal cancer and lately leukemia [27, 39-46]. The functions of miR-486-5p in cancer cells are controversial, and other reports show that miR-486-5p rather play a causative, oncogenic role, as in other solid tumours like gliomas, prostate and cervical cancer, through negative regulation of multiple tumour suppressor pathways [47-49].

There were a couple limitations to our study. The sample size of the normal samples is smaller than the included osteosarcoma samples. Furthermore, the manuscripts only showed that the hypermethylation in the differentially methylated region correlated with downregulation of miR-486-5p expression. Although a direct effect is not proven, an inverse pattern between methylation and gene expression is shown and the other results performed support the observation that the miRNA is epigenetic regulated. Further investigations regarding the epigenetic regulation of mir-486 in various tissue types would be highly interesting.

In conclusion, the present study of miR-486-5p shows that the low expression observed in osteosarcoma is caused by cancer-specific methylation of the upstream promoter region of $A N K 1$ variant 1-4. This implies a tumor suppressive role of miR-486-5p in osteosarcoma, and the findings may lead to clarify the tissue/diseasespecific regulation of the miR-486-5p expression. The main effect of miR-486-5p overexpression was morphological changes in osteosarcoma cells. The epigenetic regulation should be further correlated with clinical 
characteristics, and the functional role needs to be further validated in extended model systems.

\section{Materials and methods \\ Osteosarcoma cell lines, xenografts, patient samples and normal controls}

A panel of human osteosarcoma cell lines $(n=21)$ composed of 143B, CAL-72, G-292, HAL, HOS, IOR/MOS, IOR/OS9, IOR/OS10, IOR/OS14, IOR/OS15, IOR/ OS18, IOR/SARG, KPD, MG-63, MHM, MNNG/HOS, OHS, OSA, Saos-2, U-2 OS and ZK-58 were obtained from ATCC (www.lgcstandards-atcc.org), DSMZ (http:// www.dsmz.de/) or research partners in the EU funded EuroBoNeT project [50]. These cell lines have been extensively characterised at the molecular and phenotypic level $[10,51]$ and authenticated through STR testing.

The xenograft cohort comprised osteosarcoma xenografts established at the Norwegian Radium Hospital $(n=19)$ and at the University of Valencia $(n=22)$ [52, $53]$. In short, human tumours were implanted subcutaneously in nude mice and passed successively. These xenografts have previously shown to be good representative models for osteosarcoma, showing gene expression profiles similar to their original tumour [54].

The osteosarcoma patient sample panel comprised fresh-frozen tissue from 14 high-grade osteosarcoma samples collected at the Norwegian Radium Hospital. The tumours were diagnosed by an osteosarcoma pathologist, according to the current World Health Organization classification [55].

Six bone samples were used as normal controls. Normal bones were purchased from Capital Biosciences (Capital Biosciences, MD, USA) $(n=2)$ or obtained from amputations of cancer patients at the Norwegian Radium Hospital $(n=4)$, where bone samples were collected distant from the tumour margin. DNA copy number analysis of the latter four samples (Bone 1-4) showed normal diploid karyotype [10].

\section{miRNA expression profiling}

Total RNA from osteosarcoma cell lines and bones was extracted and quality controlled as previously described [24]. miRNA expression profiling was performed using the Agilent miRNA Complete Labeling and Hyb Kit Version 2.0, and Agilent Human miRNA Microarrays (version 2, 799 human miRNAs). miRNA data was imported into GeneSpring GX10 (Agilent Technologies Inc., CA, USA), and the intensity values were $\log _{2}$ transformed and quantile normalized. MIAME (minimum information about a microarray experiment) compliant data can be downloaded from the GEO repository (www.ncbi.nlm. nih.gov/geo/), accession number GSE28425.

\section{DNA methylation profiling}

DNA was isolated using the Wizard Genomic DNA Purification Kit (Promega, WI, USA). For the initial analyses, DNA methylation profiling of 19 cell lines and four bone samples was performed using the Illumina HumanMethylation27 BeadChip (Illumina Inc., California, USA), covering 27,000 CpG sites across the genome [10]. For an extended validation, DNA methylation profiling was performed on 10 additional patient samples and four bone samples using the Infinium HumanMethylation450 BeadChip from Illumina, covering 485,000 CpG sites across the genome. Data extraction and initial quality control of the bead summary raw data were performed using GenomeStudio V2011.1 and the Methylation module v1.9.0, both provided by Illumina. For each sample, Beta values for each probe (average ratio of signal from methylated probe relative to the sum of both methylated and unmethylated probes) were exported for downstream analysis.

\section{Integration of miRNA and methylation data}

The association between CpG methylation and miRNA expression data in osteosarcomas and normal samples was calculated using Pearson's Correlation between miR486-5p expression and methylation level (Beta) using the Methylation module v1.9.0 of GenomeStudio. DeltaBeta values for the groups of samples were calculated (Beta for cell lines minus Beta for normal samples).

\section{Quantitative real-time reverse-transcription PCR}

qRT-PCR was performed using the ABI PRISM 7500 DNA Sequence Detection System (Life Technologies, CA, USA). TaqMan MicroRNA Reverse Transcription Kit and TaqMan MiRNA Assays (Life Technologies) were used to generate cDNA and to quantitatively detect the expression of mature miR-486-5p. For pri-miRNA hsa-mir-486-1 and ANK1 expression quantification, the Fast Cells-to-Ct reagents (Life Technologies) were used to generate cDNA. Transcript-specific TaqMan Gene Expression Assays (Life Technologies) were used for quantitative PCR. Gene expression was normalized towards RNU44 for miRNA quantification and glyceraldehyde 3-phosphate dehydrogenase (GAPDH) for pri-miRNA hsa-mir-486-1 and $A L K 1$ quantification. Overview of TaqMan assays are found in Additional file 1: Supplementary Table S1. The relative expression levels were determined using the comparative $\mathrm{C}_{\mathrm{T}}$ method.

\section{5-Aza-2'-deoxycytidine treatment}

Osteosarcoma cells were cultured in the presence of $1 \mu \mathrm{M}$ 5-Aza (Sigma-Aldrich MO, USA), as previously 
described [10]. The cells were harvested after $72 \mathrm{~h}$ and total RNA was isolated using the miRNeasy Mini Kit (Qiagen, Germany).

\section{DNA extraction and bisulfite conversion}

Genomic DNA from tumour and normal samples was isolated by standard phenol chloroform extraction or using the Wizard Genomic DNA Purification Kit (Promega). DNA $(1.3 \mu \mathrm{g})$ was bisulfite treated using the EpiTect Bisulfite Kit (Qiagen) and purified using the QIAcube (Qiagen).

Qualitative methylation-specific polymerase chain reaction Primers for MSP were designed using Methyl Primer Express 1.0 (Life Technologies) (Additional file 1: Supplementary Table S2). The MSP was carried out using approximately $24 \mathrm{ng}$ of bisulfite treated DNA and performed as previously described [56]. All results were verified with a second round of MSP and scored independently by two of the authors.

\section{Direct DNA bisulfite sequencing}

A subset of the osteosarcoma cell lines was subjected to direct bisulfite sequencing as previously described [57], allowing for a semi-quantitative visualization of 5 -methylcytosines. The mir-486 primers were designed using the Methyl Primer Express 1.0 (Life Technologies) and flanked the MSP amplicons (Additional file 1: Supplementary Table S2). The approximate degree of methylation at each $\mathrm{CpG}$ site was calculated by comparing the peak height of the cytosine signal to the sum of cytosine and thymine peak height signals as previously described [58]. CpG sites with ratios between 0 and 0.2 were classified as unmethylated, CpG sites with ratios in the range of $0.21-0.8$ were classified as partially methylated, and CpG sites with ratios from 0.81-1.0 were classified as hypermethylated.

\section{Quantitative methylation-specific polymerase chain reaction}

A quantitative qMSP analysis was carried out using approximately $30 \mathrm{ng}$ of bisulfite-treated DNA and performed as previously described [56]. Commercially available fully methylated DNA (CpGenome Universal Methylated DNA, MA, USA), unconverted and bisulfite-treated normal DNA were included as controls. Primers and probes were designed using Primer Express Software 3.0 (Life Technologies) (Additional file 1: Supplementary Table S2). The $A L U$-C4 repetitive element was used as an internal reference, and the values were calculated as percent of methylated reference, PMR [59]. The median gene: Alu ratio of each gene was divided by the median gene: Alu ratio of the positive control and multiplied by 100 . To ensure high specificity for each qMSP assay, the thresholds for scoring the osteosarcoma samples as methylated were set according to the highest PMR value across the test series of normal bone. Samples with PMR values equal to or above the scoring threshold were considered to be methylated.

\section{Transfection with synthetic miRNAs and live cell imaging} Cells were seeded at a density of $1250-2500$ cells per well in 96-well plates the day before transfection. Synthetic miR-486-5p mimics and Negative Control \#1 (PM10546 and AM17110, respectively, Life Technologies) were transiently transfected into osteosarcoma cells at a final concentration of $15 \mathrm{nM}$ using the INTERFERin siRNA transfection reagent (Polyplus-transfection SA, France). The cellular growth after transfection was measured every $3 \mathrm{~h}$, by a live-cell imaging system, IncuCyte ZOOM (Essen Bioscience, UK) and the percent of cells confluence was calculated with the corresponding software application (version 2013BRev1).

\section{Statistics}

Analyses were performed with the GraphPad Prism 9 software. Non-parametric Mann-Whitney U test was applied to compare groups of samples. $p$-values were derived from two-sided tests using a significance level of 0.05 . Similarities between samples were calculated using Pearson's correlation (r). Statistical differences between the group of cell lines before and after 5-Aza treatment were calculated using a paired t-test.

\section{Supplementary Information}

The online version contains supplementary material available at https://doi. org/10.1186/s12864-022-08346-6.

Additional file 1: Supplementary Table S1. Overview of TaqMan assays for miRNA and mRNA qRT-PCR experiments. Supplementary Table S2. Primers used for qualitative Methylation-Specific Polymerase Chain Reaction (MSP), bisulfite sequencing (BS) and quantitative Methylation-Specific Polymerase Chain Reaction (qMSP). Supplementary Figure S1. Genomic overview of the mir-486/ANK1 locus. Supplementary Figure S2. Expression of ANK1 transcript variants in untreated and 5-Aza treated osteosarcoma cell lines. Supplementary Figure S3. Methylation level of miR-486 as assessed by methylation specific PCR (MSP).

Acknowledgements

We appreciate the help of Thea C. Smedsrud (Oslo University Hospital Genomics Core Facility) for performing the Infinium HumanMethylation450 BeadChip experiments.

\section{Authors' contributions}

HMN, M. Skårn, IG, DA, KA, SHK and EM performed the experiments. HMN, M. Skårn, DA, EM, GEL and LAMZ carried out experimental analyses and data interpretation. HMN, M. Skårn, GEL and LAMZ contributed to the study design. HMN and M. Skårn drafted the manuscript. OM, M. Serra, KS and ALB provided osteosarcoma samples. LAMZ and OM conceived of the study. All authors read and approved the final manuscript. 


\section{Funding}

The study was funded by EuroBoNet, a European Commission granted Network of Excellence for studying the pathology and genetics of bone tumours (LSHC-CT-2006-018814), the Norwegian Cancer Society grant number PR-2007-0163 and the Ragnvarda F. Sørvik and Håkon Starheims legacy. No potential conflicts of interest were disclosed.

\section{Availability of data and materials}

For the microarray experiments, MIAME compliant data can be downloaded from the GEO data repository (www.ncbi.nlm.nih.gov/geo/). The Agilent Human miRNA Microarray datasets are deposited under the accession number GSE28425, the Illumina HumanMethylation27 datasets under the accession number GSE36002 in SuperSeries number GSE36004 and the Infinium HumanMethylation450 DNA methylation datasets under the accession number GSE161407.

\section{Declarations}

\section{Ethics approval and consent to participate}

For the human samples, all methods were carried out in accordance with relevant guidelines and regulations. The patients included have diseased, and according to the regulations a consent to use is not required, and the project and the collection of samples were approved by the Ethical Committee of Southern Norway (Project 17866).

Xenograft experiments were performed according to protocols approved by the Norwegian Animal Research Authority and the Institutional Animal Care of Valencia University. Animal experiments were followed protocols and guidelines approved by the National Animal Research Authority in compliance with the European Convention of the Protection of Vertebrates Used for Scientific Purposes (approval ID 10175) and were approved by Oslo University Hospital. All methods were performed and reported in accordance with ARRIVE guidelines.

\section{Consent for publication}

Not applicable.

\section{Competing interests}

The authors declare that they have no competing interests.

\section{Author details}

1 Department of Tumor Biology, Institute for Cancer Research, The Norwegian Radium Hospital, Oslo University Hospital, Oslo, Norway. ${ }^{2}$ Department of Molecular Oncology, Institute for Cancer Research, The Norwegian Radium Hospital, Oslo University Hospital, Oslo, Norway. ${ }^{3}$ Laboratory of Experimental Oncology, IRCCS Istituto Ortopedico Rizzoli, Bologna, Italy. ${ }^{4}$ Department of Pathology, Valencia University, Valencia, Spain. ${ }^{5}$ Department for Clinical Science, University of Bergen, Bergen, Norway. ${ }^{6}$ Genomics Core Facility, Department of Core Facilities, Institute for Cancer Research, The Norwegian Radium Hospital, Oslo University Hospital, Oslo, Norway.

Received: 21 April 2021 Accepted: 27 January 2022 Published online: 17 February 2022

\section{References}

1. Friebele JC, Peck J, Pan X, Abdel-Rasoul M, Mayerson JL. Osteosarcoma: a Meta-analysis and review of the literature. Am J Orthop (Belle Mead NJ). 2015;44(12):547-53.

2. Hattinger CM, Patrizio MP, Magagnoli F, Luppi S, Serra M. An update on emerging drugs in osteosarcoma: towards tailored therapies? Expert Opin Emerg Drugs. 2019;24(3):153-71.

3. Perry JA, Kiezun A, Tonzi P, Van Allen EM, Carter SL, Baca SC, et al. Complementary genomic approaches highlight the PI3K/mTOR pathway as a common vulnerability in osteosarcoma. Proc Natl Acad Sci. 2014;111(51):E5564.

4. Kovac M, Blattmann C, Ribi S, Smida J, Mueller NS, Engert F, et al. Exome sequencing of osteosarcoma reveals mutation signatures reminiscent of BRCA deficiency. Nat Commun. 2015;6:8940.
5. Lorenz S, Baroy T, Sun J, Nome T, Vodak D, Bryne JC, et al. Unscrambling the genomic chaos of osteosarcoma reveals extensive transcript fusion, recurrent rearrangements and frequent novel TP53 aberrations. Oncotarget. 2016;7(5):5273-88.

6. Behjati S, Tarpey PS, Haase K, Ye H, Young MD, Alexandrov LB, et al. Recurrent mutation of IGF signalling genes and distinct patterns of genomic rearrangement in osteosarcoma. Nat Commun. 2017;8:15936.

7. Hattinger CM, Patrizio MP, Luppi S, Serra M. Pharmacogenomics and Pharmacogenetics in osteosarcoma: translational studies and clinical impact. Int J Mol Sci. 2020;21(13):4659.

8. Hattinger CM, Patrizio MP, Tavanti E, Luppi S, Magagnoli F, Picci P, et al. Genetic testing for high-grade osteosarcoma: a guide for future tailored treatments? Expert Rev Mol Diagn. 2018;18(11):947-61.

9. Sadikovic B, Yoshimoto M, Chilton-Macneill S, Thorner P, Squire JA, Zielenska M. Identification of interactive networks of gene expression associated with osteosarcoma Oncogenesis by integrated molecular profiling Hum Mol Genet. 2009;18(11):1962-75.

10. Kresse SH, Rydbeck H, Skårn M, Namløs HM, Barragan-Polania AH, CletonJansen A-M, et al. Integrative analysis reveals relationships of genetic and epigenetic alterations in osteosarcoma. PLoS One. 2012;7(11):e48262.

11. Mills LJ, Scott MC, Shah P, Cunanan AR, Deshpande A, Auch B, et al. Comparative analysis of genome-wide DNA methylation identifies patterns that associate with conserved transcriptional programs in osteosarcoma. Bone. 2020:115716.

12. Shu J, Li L, Sarver AE, Pope EA, Varshney J, Thayanithy V, et al. Imprinting defects at human 14q32 locus alters gene expression and is associated with the pathobiology of osteosarcoma. Oncotarget. 2016;7(16):21298-314.

13. Asano N, Takeshima H, Yamashita S, Takamatsu H, Hattori N, Kubo T, et al. Epigenetic reprogramming underlies efficacy of DNA demethylation therapy in osteosarcomas. Sci Rep. 2019;9(1):20360.

14. Li B, Ye Z. Epigenetic alterations in osteosarcoma: promising targets. Mol Biol Rep. 2014:41(5):3303-15.

15. Kulis M, Esteller M. DNA methylation and cancer. Adv Genet. 2010;70:27-56.

16. Berdasco M, Esteller M. Clinical epigenetics: seizing opportunities for translation. Nat Rev Genet. 2019;20(2):109-27.

17. Michalak EM, Burr ML, Bannister AJ, Dawson MA. The roles of DNA, RNA and histone methylation in ageing and cancer. Nat Rev Mol Cell Biol. 2019;20(10):573-89.

18. Saxonov S, Berg P, Brutlag DL. A genome-wide analysis of CpG dinucleotides in the human genome distinguishes two distinct classes of promoters. Proc Natl Acad Sci. 2006;103(5):1412.

19. Suzuki MM, Bird A. DNA methylation landscapes: provocative insights from epigenomics. Natl Rev. 2008;9(6):465-76.

20. Yao $Q$, Chen $Y$, Zhou $X$. The roles of microRNAs in epigenetic regulation. Curr Opin Chem Biol. 2019;51:11-7.

21. Weber B, Stresemann C, Brueckner B, Lyko F. Methylation of human microRNA genes in normal and neoplastic cells. Cell Cycle. 2007;6(9):1001-5.

22. Maire G, Martin JW, Yoshimoto M, Chilton-MacNeill S, Zielenska M, Squire JA. Analysis of miRNA-gene expression-genomic profiles reveals complex mechanisms of microRNA deregulation in osteosarcoma. Cancer Genetics. 2011;204(3):138-46.

23. Jones KB, Salah Z, Sara DM, Galasso M, Gaudio E, Nuovo GJ, et al. MicroRNA signatures associate with pathogenesis and progression of osteosarcoma. Cancer Res. 2012;72(7):1865-77.

24. Namløs HM, Meza-Zepeda LA, Barøy T, Østensen IHG, Kresse SH, Kuijjer $\mathrm{ML}$, et al. Modulation of the osteosarcoma expression phenotype by MicroRNAs. PLoS One. 2012;7(10):e48086.

25. Lietz CE, Garbutt C, Barry WT, Deshpande V, Chen YL, Lozano-Calderon SA, et al. MicroRNA-mRNA networks define translatable molecular outcome phenotypes in osteosarcoma. Sci Rep. 2020;10(1):4409.

26. He M, Wang G, Jiang L, Qiu C, Li B, Wang J, et al. miR-486 suppresses the development of osteosarcoma by regulating PKC- $\delta$ pathway. Int J Oncol. 2017;50(5):1590-600.

27. Tessema M, Yingling CM, Picchi MA, Wu G, Ryba T, Lin Y, et al. ANK1 methylation regulates expression of MicroRNA-486-5p and discriminates lung tumors by histology and smoking status. Cancer Lett. 2017;410:191-200. 
28. Gallagher PG, Forget BG. An alternate promoter directs expression of a truncated, muscle-specific isoform of the human ankyrin 1 gene. J Biol Chem. 1998;273(3):1339-48.

29. Gallagher PG, Romana M, Tse WT, Lux SE, Forget BG. The human ankyrin-1 gene is selectively transcribed in erythroid cell lines despite the presence of a housekeeping-like promoter. Blood. 2000;96(3):1136-43.

30. Baskerville S, Bartel DP. Microarray profiling of microRNAs reveals frequent coexpression with neighboring miRNAs and host genes. RNA. 2005:11(3):241-7.

31. Hinske LCG, Galante PAF, Kuo WP, Ohno-Machado L. A potential role for intragenic miRNAs on their hosts'interactome. BMC Genomics. 2010;11:533.

32. Jacox E, Gotea V, Ovcharenko I, Elnitski L. Tissue-specific and ubiquitous expression patterns from alternative promoters of human genes. PLoS One. 2010;5(8):e12274.

33. Bennett $V$, Lorenzo DN. Spectrin- and ankyrin-based membrane domains and the evolution of vertebrates. Curr Top Membr. 2013;72:1-37.

34. Lunnon K, Smith R, Hannon E, De Jager P, Srivastava G, Volta M, et al. Cross-tissue methylomic profiling strongly implicates a role for cortexspecific deregulation of ANK1 in Alzheimer's disease neuropathology. Nat Neurosci. 2014;17(9):1164-70.

35. Shaham L, Vendramini E, Ge Y, Goren Y, Birger Y, Tijssen MR, et al. MicroRNA-486-5p is an erythroid oncomiR of the myeloid leukemias of Down syndrome. Blood. 2015;125(8):1292-301.

36. Small EM, O'Rourke JR, Moresi V, Sutherland LB, McAnally J, Gerard RD, et al. Regulation of PI3-kinase/Akt signaling by muscle-enriched microRNA-486. Proc Natl Acad Sci U S A. 2010;107(9):4218-23.

37. Liu Y, Zhang J, Xing C, Wei S, Guo N, Wang Y. miR-486 inhibited osteosarcoma cells invasion and epithelial-mesenchymal transition by targeting PIM1. Cancer Biomarkers. 2018;23:269-77.

38. Song R, Fullerton DA, Ao L, Ks Z, Reece TB, Cleveland JC, et al. Altered MicroRNA expression is responsible for the pro-Osteogenic phenotype of interstitial cells in calcified human aortic valves. J Am Heart Assoc. 2017;6(4):e005364.

39. Liu H, Ni Z, Shi L, Ma L, Zhao J. MiR-486-5p inhibits the proliferation of leukemia cells and induces apoptosis through targeting FOXO1. Mol Cell Probes. 2019;44:37-43.

40. Oh HK, Tan AL, Das K, Ooi CH, Deng NT, Tan IB, et al. Genomic loss of miR486 regulates tumor progression and the OLFM4 Antiapoptotic factor in gastric Cancer. Clin Cancer Res. 2011;17(9):2657-67.

41. Borjigin N, Ohno S, Wu W, Tanaka M, Suzuki R, Fujita K, et al. TLS-CHOP represses miR-486 expression, inducing upregulation of a metastasis regulator PAI-1 in human myxoid liposarcoma. Biochem Biophys Res Commun. 2012:427:355-60.

42. Peng $Y$, Dai $Y$, Hitchcock C, Yang $X$, Kassis ES, Liu L, et al. Insulin growth factor signaling is regulated by microRNA-486, an underexpressed microRNA in lung cancer. Proc Natl Acad Sci. 2013;110(37):15043-8.

43. Zhang G, Liu Z, Cui G, Wang X, Yang Z. MicroRNA-486-5p targeting PIM-1 suppresses cell proliferation in breast cancer cells. Tumor Biol. 2014;35(11):11137-45.

44. Liu X, Chen X, Zeng K, Xu M, He B, Pan Y, et al. DNA-methylation-mediated silencing of miR-486-5p promotes colorectal cancer proliferation and migration through activation of PLAGL2/IGF2/ $\beta$-catenin signal pathways. Cell Death Dis. 2018;9(10):1037.

45. Lang B, Zhao S. miR-486 functions as a tumor suppressor in esophageal cancer by targeting CDK4/BCAS2. Oncol Rep. 2018;39(1):71-80.

46. Huang $X-P$, Hou J, Shen $X-Y$, Huang $C-Y$, Zhang $X-H$, Xie $Y-A$, et al. MicroRNA-486-5p, which is downregulated in hepatocellular carcinoma, suppresses tumor growth by targeting PIK3R1. FEBS J. 2015;282(3):579-94.

47. Tetzlaff MT, Curry JL, Yin V, et al. Dlstinct pathways in the pathogenesis of sebaceous carcinomas implicated by differentially expressed micrornas. JAMA Ophthalmol. 2015;133:1109-16.

48. Yang Y, Ji C, Guo S, Su X, Zhao X, Zhang S, et al. The miR-486-5p plays a causative role in prostate cancer through negative regulation of multiple tumor suppressor pathways. Oncotarget. 2017;8(42):72835-46.

49. Li C, Zheng X, Li W, Bai F, Lyu J, Meng QH. Serum miR-486-5p as a diagnostic marker in cervical cancer: with investigation of potential mechanisms. BMC Cancer. 2018;18:61.

50. Ottaviano L, Schaefer KL, Gajewski M, Huckenbeck W, Baldus S, Rogel U, et al. Molecular characterization of commonly used cell lines for bone tumor research: a trans-European EuroBoNet effort. Genes Chromosomes Cancer. 2010;49(1):40-51.

51. Lauvrak SU, Munthe E, Kresse SH, Stratford EW, Namlos HM, Meza-Zepeda LA, et al. Functional characterisation of osteosarcoma cell lines and identification of mRNAs and miRNAs associated with aggressive cancer phenotypes. Br J Cancer. 2013;109(8):2228-36.

52. Mayordomo E, Machado I, Giner F, Kresse SH, Myklebost O, Carda C, et al. A tissue microarray study of osteosarcoma: histopathologic and immunohistochemical validation of xenotransplanted tumors as preclinical models. Appl Immunohistochem Mol Morphol. 2010;18(5):453-61.

53. Kresse SH, Meza-Zepeda LA, Machado I, Llombart-Bosch A, Myklebost O. Preclinical xenograft models of human sarcoma show nonrandom loss of aberrations. Cancer. 2012;118(2):558-70.

54. Kuijjer ML, Namlos HM, Hauben El, Machado I, Kresse SH, Serra M, et al. mRNA expression profiles of primary high-grade central osteosarcoma are preserved in cell lines and xenografts. BMC Med Genet. 2011;4:66.

55. Raymond AK, Ayala AG, Knuutila S. Conventional osteosarcoma. In: Fletcher CDM, Unni KK, Mertens F, editors. World Health Organization classification of Tumours pathology and genetics of Tumours of soft tissue and bone. Lyon: IARC Press; 2002.

56. Lind GE, Danielsen SA, Ahlquist T, Merok MA, Andresen K, Skotheim RI, et al. Identification of an epigenetic biomarker panel with high sensitivity and specificity for colorectal cancer and adenomas. Mol Cancer. 2011;10:85.

57. Lind GE, Ahlquist T, Kolberg M, Berg M, Eknaes M, Alonso MA, et al. Hypermethylated MAL gene - a silent marker of early colon tumorigenesis. J Transl Med. 2008;6:13

58. Melki JR, Vincent PC, Clark SJ. Concurrent DNA hypermethylation of multiple genes in acute myeloid leukemia. Cancer Res. 1999;59(15):3730-40.

59. Widschwendter M, Siegmund KD, Muller HM, Fiegl H, Marth C, MullerHolzner $E_{1}$ et al. Association of breast cancer DNA methylation profiles with hormone receptor status and response to tamoxifen. Cancer Res. 2004;64(11):3807-13.

\section{Publisher's Note}

Springer Nature remains neutral with regard to jurisdictional claims in published maps and institutional affiliations.

Ready to submit your research? Choose BMC and benefit from

- fast, convenient online submission

- thorough peer review by experienced researchers in your field

- rapid publication on acceptance

- support for research data, including large and complex data types

- gold Open Access which fosters wider collaboration and increased citations

- maximum visibility for your research: over 100M website views per year

At BMC, research is always in progress.

Learn more biomedcentral.com/submissions 